\title{
PROCESSO DE CONSTRUÇÃO DAS LINHAS DE CUIDADO EM SAÚDE NA ESTRATÉGIA SAÚDE DA FAMÍLIA
}

\author{
CONSTRUCTION PROCESS OF THE HEALTH CARE LINES IN FAMILY HEALTH \\ STRATEGY \\ Meire Núbia Santos Santana, Adélia Maria Carvalho De Melo Pinheiro,Regina Lúcia Almeida \\ Lino Vieira, Rozemere Cardoso De Souza
}

Universidade Estadual De Santa Cruz - UESC

\section{Abstract}

The objective of this study was to analyze the meanings given to the process of construction of the care lines in the Family Health Units of the municipality of Ilhéus, Bahia, by participants of the Education for Work Program for Health, in the city of Ilhéus, Bahia. This is a qualitative research, carried out in 12 units, with 77 participants, including health professionals, students and teachers. Data were collected between the years of 2010 to 2012, through focus groups, field diary and survey of action research plans. The analysis of the materials occurred from the interweaving between identified thematic categories, theoretical reference and interpretation of the researchers. The results were described based on the following themes: a) trajectories of choice of population groups and stages of construction of the health care lines of each unit; b) perceptions about the moment of greatest relevance, characterized by the description of the prominent moments for each team; c) facilities and difficulties experienced. It was concluded that the Program served as a tool to assist the process of construction of care lines, involved intervention actions and research aimed at different population groups according to the daily experiences of the teams investigated. In addition, this process has also strengthened health workers in the face of limits and in the search for overcoming the difficulties that have arisen during the organization of services.

Keywords: Family Health; Health Human Resource Training; Integrality in Health.

\section{Resumo}

Este estudo teve por objetivo geral analisar os sentidos dados ao processo de construção das linhas de cuidado nas Unidades de Saúde da Família do município de Ilhéus, Bahia, por participantes do Programa de Educação pelo Trabalho para a Saúde, no município de Ilhéus, Bahia. Trata-se de pesquisa qualitativa, realizada em 12 unidades, com 77 participantes, incluindo profissionais de saúde, estudantes e professores. Os dados foram coletados entre os anos de 2010 a 2012, através de grupos focais, diário de campo e de levantamento dos planos de pesquisa-ação. A análise dos materiais ocorreu a partir do entrelaçamento entre categorias temáticas identificadas, referencial teórico e interpretação das pesquisadoras. Os resultados foram descritos a partir dos seguintes temas: a) trajetórias de escolha dos grupos populacionais e de etapas de construção das linhas de cuidado em saúde de cada unidade; b) percepções acerca do momento de maior relevância, caracterizadas pela descrição dos momentos proeminentes para cada equipe; c) facilidades e dificuldades vivenciadas. Conclui-se que o Programa serviu de ferramenta para auxiliar o processo de construção de linhas de cuidado, envolveu ações de intervenção $e$ pesquisas voltadas para diversos grupos populacionais de acordo com as vivências do cotidiano profissional das equipes investigadas. Além disso, esse processo também fortaleceu trabalhadores da saúde no enfrentamento dos limites e na busca de superação das dificuldades surgidas nas etapas de organização dos serviços.

Palavras-chave: Saúde da Família; Capacitação de recursos humanos em saúde; Assistência integral à saúde. 
Introdução

O contexto de reorientação do modelo assistencial em saúde requer, através da Estratégia Saúde da Família (ESF), mudanças no processo de trabalho em saúde, com ênfase na produção do cuidado, incluindo participação social, exercício da integralidade, dentre outros princípios do Sistema Único de Saúde (SUS). A prestação de serviços deve ocorrer de maneira a utilizar tecnologias diferentes e adequadas às necessidades apresentadas pelo indivíduo e pela coletividade. Assim, com tecnologias apropriadas, o sistema funcionaria baseado na referência e contrarreferência, visando integralidade e produção do cuidado em saúde ${ }^{1}$.

O cuidado é visto como satisfação da necessidade do outro e pode ser produzido por duas modalidades distintas: incentivando ou impedindo a autonomia das pessoas. Na primeira situação, ocorrem atitudes de incentivo à autonomia e facilitação da participação das pessoas nas decisões relativas ao cuidado, reconhecendo-as como sujeitos da própria saúde. No segundo caso, a produção do cuidado não estimula a autonomia das pessoas, sendo constitutivo de atitudes de superproteção que dificultam os processos de promoção da saúde e reabilitação ${ }^{2}$.

Contudo, no cotidiano dos serviços, percebe-se permanência no agir em saúde, caracterizado mais como produção de procedimentos do que como produção de cuidados. A ênfase na produção de procedimentos implica um tipo de gestão que inclui oferta de consultas, curativos, vacinas e outros procedimentos, sem assumir compromissos com os usuários e suas necessidades ${ }^{3}$. Tais processos produtivos centrados nos procedimentos voltam-se mais para equipamentos e tecnologias duras do que propriamente para aquilo que seria sua finalidade, a de produzir cuidados para que as pessoas vivam melhor suas vidas ${ }^{4}$.

Nesse contexto, surge a necessidade de desenvolvimento de linhas de cuidado como proposições organizativas dos serviços para vencer os desafios na direção da produção de cuidado integral ${ }^{5}$. Linhas de cuidado configuramse como processo de construção do cuidado, situado no tempo e contexto de determinado território ou serviço, e envolvem decisões/ intervenções planejadas, acerca das prioridades em saúde de indivíduos ou grupo de pessoas que guardem semelhanças quanto ao perfil sociodemográfico (ciclos de vida) ou problemas de saúde prioritários. Assim, tal processo é dinâmico, constitutivo da rede formal e informal (autocuidado e manejo comunitário) de práticas e tecnologias de uso para prevenção, recuperação, reabilitação e promoção da saúde ${ }^{6}$.

Tomando como base essas considerações, as pesquisadoras passaram a refletir a experiência do Programa de Educação pelo Trabalho para a Saúde (PET-Saúde) em Ilhéus, Bahia. Esse Programa surgiu como meio de contribuição para estruturação das linhas de cuidado em Unidades de Saúde da Família (USF's), com as equipes da ESF, docentes e estudantes de uma universidade pública estadual da Bahia. Questionou-se sobre: como ocorreram as etapas e ações de estruturação de linhas de cuidado, a partir das equipes PET-Saúde do município de Ilhéus, Bahia? Como essas equipes explicam a escolha de um grupo populacional para construção da linha de cuidado? Quais as intervenções e ações desenvolvidas por elas, frente aos problemas identificados? Quais as facilidades e dificuldades desse processo?

Assim, este estudo teve como objetivo geral analisar os sentidos dados ao processo de construção das linhas de cuidado nas USF's do município de Ilhéus, Bahia, por participantes do Programa de Educação pelo Trabalho para a Saúde. E como objetivos específicos: caracterizar os caminhos para a construção das linhas de cuidado nessas USF's; identificar intervenções produzidas por esses trabalhadores nesses grupos populacionais específicos; descrever facilidades e dificuldades do processo de construção das linhas de cuidado.

A relevância deste estudo está na possibilidade de contribuir com ações de integração ensino-serviço, de (re)orientações acerca do modo de organizar o processo de trabalho em saúde com impacto na formação e na qualificação de profissionais de saúde comprometidos com os princípios do SUS, especialmente com a integralidade da atenção em saúde. Busca também contribuir com as reflexões acerca da transformação de práticas tradicionais em práticas cuidadoras e integrais no contexto de atuação da ESF.

\section{Metodologia}

Este estudo orientou-se a partir de pressupostos da pesquisa qualitativa e construcionista social, nos quais a produção de conhecimento baseia-se pela reflexibilidade de determinados fenômenos, atenta à pluralidade de significados, afetos, criatividade e posicionamentos sociais, dentro de um campo específico e espaço temporal ${ }^{7,8}$. 
O construcionismo, conforme abordagem da Psicologia social, define o conhecimento como produto das relações entre as pessoas, portanto, da linguagem em uso no cotidiano. Reflete, pois, a multiplicidade de sentidos produzidos em diferentes práticas discursivas, a pluralidade de saberes e vozes de atores sociais presentes ou presentificados nesses discursos e como as pessoas se posicionam nas interações do cotidiano em relação aos sentidos dados ao fenômeno estudado ${ }^{7}$.

Nesse sentido, pode-se pensar a produção de conhecimento à luz desse referencial teóricometodológico como possibilidade para refletir a saúde e a produção de cuidado como processos em constante mudança, criativos e inovadores, ainda que se identifiquem permanências, nos saberes e práticas produzidos no cotidiano.

0 trabalho foi desenvolvido em 12 áreas de abrangência de equipes das USF's participantes do PET-Saúde da cidade de Ilhéus, Bahia, a saber: Nossa Senhora da Vitória I, II e III, Teotônio Vilela I, II, III, IV, Salobrinho I e II, Barra e Basílio I e II.

Participaram do estudo 77 membros do PET-Saúde, sendo 24 profissionais da Unidade de Saúde da Família, 48 estudantes bolsistas e 5 tutores acadêmicos, que atenderam aos seguintes critérios de inclusão: atuar desde o ano de 2009, ano de implantação do Programa em Ilhéus; ser eleito pela equipe PET-Saúde para representá-la no estudo, na condição de preceptor ou de estudante bolsista do Programa. O critério de exclusão foi: não ter feito parte do Programa desde 2009.

A coleta de dados ocorreu no período de 2010 a 2012. Utilizou-se a técnica de grupo focal, ferramenta que permitiu aos sujeitos sociais expressarem percepções, crenças e valores, assim como os sentidos dados aos temas discutidos. No grupo, ocorreu processo de interação onde os comentários de um estimulavam a fala de outros, onde o silêncio era sempre interrompido.

Foram realizados dois grupos focais de sessão única, em salas de uma universidade pública, reservadas para este fim, com atenção à preservação da privacidade, e à prevenção de interrupções ou de ruídos do ambiente que pudessem prejudicar os diálogos.

Os grupos tiveram duração média de duas horas, a partir de três momentos essenciais: a) preparação, com apresentação dos participantes, de forma lúdica, das informações complementares acerca da dinâmica e esclarecimento de dúvidas; b) discussão, orientada por um guia de temas, construída previamente, contendo questões relacionadas aos objetivos do estudo. Nesse momento, também foram construídos painéis que representassem as escolhas dos grupos populacionais de cada equipe para estruturação das linhas de cuidado, e foi mantida flexibilidade para possibilitar a introdução de questões, pertinentes aos objetivos da pesquisa, que não estavam contempladas no guia de temas; c) fechamento, que consistiu no resumo dos conteúdos dos grupos, nas anotações provenientes dos repertórios e das expressões não verbais, sem ajuizar sobre as diferenças de opinião entre os participantes, e de agradecimentos.

Os grupos foram moderados por docentes da equipe técnica do projeto e por uma psicóloga que presta apoio técnico ao Grupo de Pesquisa em Saúde Mental da Universidade Estadual de Santa Cruz (UESC), todos com experiência em dinâmicas grupais. Participaram também dois observadores, responsáveis pela gravação dos conteúdos emergentes dos grupos. Estes últimos foram estudantes bolsistas do PET-Saúde em Ilhéus, recém-ingressos no Programa, sendo um do curso de Enfermagem e um do curso de Medicina, devidamente capacitados para o exercício desta atividade.

A coleta de dados deste estudo envolveu, também, registros de diários de campo decorrentes das reuniões mensais, de reflexões conjuntas na atividade tutorial e de levantamentos dos planos de pesquisa-ação construídos e desenvolvidos pelas Equipes da ESF.

Para análise dos dados, os conteúdos emergentes dos grupos foram transcritos, e foi atribuído a cada participante um código (P1, P2 e outros). As etapas de análise descritas a seguir foram orientadas a partir do enfoque construcionista social, segundo Spink ${ }^{9}$ : a) leituras exaustivas do material transcrito, b) identificação de repertórios interpretativos, c) definição de categorias temáticas; d) interpretação dos resultados obtidos, a partir do entrelaçamento entre as categorias temáticas, o referencial teórico, a literatura sobre o assunto e a interpretação destas pesquisadoras. Os conteúdos advindos do diário de campo e do levantamento dos projetos de cada equipe foram utilizados como dados complementares aos conteúdos emergentes dos grupos focais.

Repertórios interpretativos referem-se ao "conjunto identificáveis de termos, descrições, figuras de linguagem, metáforas ou imagens vividas, empregadas nas construções discursivas em diferentes tipos de interações conversacionais" 10:68. Através do uso dos 
repertórios, as pessoas dão sentidos/ significados às suas vivências, às coisas e fenômenos que lhes acontecem, e se posicionam no cotidiano ${ }^{9}$. Por isso, nessa perspectiva de análise, dizeres e fazeres são entendidos como unidades inseparáveis, o que valoriza ainda mais o uso da mesma neste estudo sobre linhas de cuidado. Como diz Rocha 11:619, "antes de representar o mundo, o discurso é um modo de intervir". Portanto, não se trata de representar o mundo ${ }^{11}$, e sim de inventá-lo e reinventá-lo, através da linguagem em uso de diversas práticas discursivas.

Esta pesquisa cumpriu os aspectos éticos da pesquisa com seres humanos e foi aprovada pelo Comitê de Ética em Pesquisa da Universidade Estadual de Santa Cruz (CEP/ UESC), sob o parecer consubstanciado no 568, de 16 de junho de 2010, cujos princípios que o fundamentam são os mesmos definidos na Resolução 466/2012 do Conselho Nacional de Saúde (CNS)/Ministério da Saúde (MS).

\section{Resultados e Discussões}

Os resultados permitiram descrever três categorias temáticas, explicativas dos sentidos atribuídos pelos participantes ao processo de construção de linhas de cuidado, no contexto da ESF e do PET-Saúde, através, dentre outros aspectos supracitados, da identificação do uso dos repertórios interpretativos nos conteúdos emergentes das falas desses participantes: a) trajetórias de escolha dos grupos populacionais e de etapas de construção das linhas de cuidado em saúde de cada unidade; b) percepções acerca do momento de maior relevância, caracterizadas pela descrição dos momentos proeminentes para cada equipe; c) facilidades e dificuldades vivenciadas.

Trajetórias de escolha dos grupos populacionais e de etapas de construção das linhas de cuidado em saúde de cada USF

Este tema reúne os repertórios explicativos dos sentidos dados às razões para escolha dos grupos populacionais alvo da construção das linhas de cuidado em saúde de cada USF e às trajetórias dessa construção.

A escolha dos grupos alvo foi o primeiro passo para o processo de estruturação das linhas de cuidado e foi definida por ciclos do desenvolvimento humano (idosos, crianças, adolescentes), gênero (homens) e toda a população adscrita do território de uma ESF, neste último caso, buscou-se desenvolver participação e controle social.

Os grupos prioritários foram escolhidos de modo singular por cada equipe, e, nas falas dos participantes, as razões estavam relacionadas aos saberes acerca de dados de perfis e das condições de saúde da população adscrita, e das vivências do cotidiano do serviço, quanto aos procedimentos e/ ou programas ofertados: saúde do idoso, Programa Hiperdia, saúde mental, vigilância epidemiológica, saúde da criança e do adolescente. Outras razões explicativas da escolha de grupos prioritários foram: identificação de ausência/lacuna de programas específicos e necessários, baixa adesão da população aos serviços existentes, e motivações dos participantes da linha de cuidado a ser construída.

Assim, o uso dos termos, como "poucos", "nenhum", "grande número", "Sistema de Informação da Atenção Básica (SIAB)", "programa", "demanda", "adolescente", deram sentidos aos saberes e práticas produzidos pelos participantes, na etapa inicial da construção da linha de cuidado, exemplificados nas falas a seguir:

"Poucos homens que frequentavam a unidade" (P9).

"Grande número de pessoas, pouca adesão ao tratamento e ao Programa Hiperdia" (P10).

"Nenhum trabalho específico; dados do SIAB; população grande de adolescentes" (P3).

"Acolhimento de adolescentes, durante o decorrer do PET, foi implementando outras atividades" (P4).

"Ouvir a população e saber da demanda. Falta de um programa para adolescente; dados do SIAB; violência e agravo" (P1).

Os repertórios interpretativos utilizados pelos participantes, para explicar recursos existentes e tecnologias aplicadas em outras etapas do processo de estruturação das linhas de cuidado aos grupos populacionais escolhidos, foram, dentre outros, "visita domiciliar", "consulta", "reuniões" e "oficinas de educação e saúde". Os recortes das falas a seguir exemplificam esses sentidos.

"Realizamos visita domiciliar aos adolescentes para convidá-los a comparecerem à Unidade de Saúde, objetivando realizar a avaliação de seu crescimento e desenvolvimento e, caso necessário, regularização de sua cobertura vacinal" (P1). 
"Fizemos consultas, encaminhamos para vacinação [...]" (P2).

"Realização de oficina Violência sexual contra crianças e adolescentes" (P3).

"Entrevistados os adolescentes e tabulados os dados coletados" (P4).

"Reunião, objetivando articulação com o Pró-jovem" (P1).

"Realizada oficina com adolescentes do Prójovem sobre sexualidade" (P2).

"Capacitação dos Agentes Comunitários de Saúde para realizar atividades na Primeira Semana Integral à Saúde da Criança" (P6).

"Oficina sobre o Envelhecimento Saudável" (P5).

"Capacitação de Equipe de Saúde da Família para acolhimento aos idosos" (P7).

"Reunião com representantes dos conselhos local e municipal de saúde, das escolas do bairro e da Secretaria de Saúde Municipal e Secretaria de Educação Municipal" (P8).

"Cadastramento dos idosos e dos cuidadores" (P5).

Em algumas das descrições supracitadas e nas trajetórias exemplificadas a seguir, como coleta de dados secundários e primários, apropriação de inúmeras variáveis explicativas dos problemas enfrentados pelos grupos, envolvimento do usuário e cartografia da realidade local, identificaram-se, ainda, que os participantes também fizeram uso da pesquisa como dispositivo para definição de ações e de serviços a serem prestados:

"Cartografia; levantamento dos dados no SIAB; homens iam mais para o Hiperdia" (P11).

"Reconhecimento da área; dados do SIAB; questionário do número de idosos de todas as microáreas; ações educativas; consultas de enfermagem; médica; cadastro" (P7).

"Questionário aplicado aos clientes, finalizando com ações educativas [...]" (P12)

"Ações educativas [...] trabalho com a equipe; dados do SIAB" (P9).

"Reconhecimento da área; dados do SIAB; pessoas que lutavam pela causa, porque buscavam o atendimento 24 horas; situação de agravo; campanha de vacina contra hepatite [...] finalizou com o fórum [...]" (P8).

"Adolescentes divididos por faixa etária [...] listagem dos problemas vividos pelos adolescentes [...] temas: dança e drogas [...] finalizou com o Fórum [...] autoavaliação do grupo PET. Sugestão, dos adolescentes, que o Fórum continuasse. Estão querendo a participação do Conselho Local de Saúde. A ideia era que o PET Saúde continuasse [...] questionário conhecendo o adolescente" (P2).

Esses conteúdos descrevem, pois, modos de produzir saúde e de se posicionar frente aos desafios para estruturação de linhas de cuidado, relacionados aos resultados de pesquisas, ao acesso ou não dos usuários aos serviços ofertados pela ESF e também ao desejo, às inquietações e ao tempo de vivências do profissional ou equipe de saúde junto à população que acompanha. Disto resultam singularidades e diversidade de escolhas dos grupos e também das tecnologias utilizadas por cada equipe.

Tais sentidos convergem para características próprias do campo da Saúde Coletiva, um campo complexo, que trata tanto do particular como do geral, heterogêneo por refletir diversos interesses, relações e tensionamentos, e multidisciplinar, estando sempre aberto aos saberes e práticas inovadoras $^{13}$.

Sobre enfrentamentos, Bertotti, Lenzi, Portes ${ }^{14}$ citam a literatura tratar do assunto como estado particular e único na vida de cada pessoa, sendo questão multimensional, mobilizadora de recursos internos, do ambiente e do tipo e da condição da morbidade que afeta esse sujeito.

Parece consenso entre os participantes a criação de espaços de aproximações e de diálogos entre eles e as pessoas alvo das práticas de cuidado, e entre estes e atores de outros serviços e programas, tais como o Pró-jovem e a Secretaria de Saúde. Valorizam-se aqui o uso do acolhimento, da escuta e do vínculo, como tecnologias em potencial para mudanças da atenção à saúde, e para criação de tessituras entre pontos de redes de cuidado ${ }^{6}$.

Aproximar pontos da rede entre serviços e programas podem reorientar relações em busca de fluxos de atendimentos às demandas $e$ necessidades de saúde, de modo articulado para atenção resolutiva. Em situações que envolvam linhas de cuidado às condições crônicas, dentre outras citadas aqui, essa articulação é necessária para continuidade das ações, num agir comprometido com a integralidade ${ }^{15}$. Assim, os sentidos aqui produzidos revelam práticas discursivas que rompem com o habitual dos serviços, para possibilidades de relações inovadoras entre os atores citados, e também entre atores do ensino e da ESF e comunidade, como foi a proposta do PET-Saúde, e pode ser a de outros programas afins. 
Percepções sobre o momento de maior relevância na construção das linhas de cuidado

Nos conteúdos grupais, os participantes descreveram como momentos proeminentes da trajetória de construção de linhas de cuidado as práticas instituídas a partir do PET-Saúde e de outras vivências acadêmicas desenvolvidas por discentes e docentes no cenário da ESF, que provocaram mudanças no processo de trabalho da equipe de saúde. Isto resultou no estreitamento da relação entre a equipe de saúde e a comunidade, e aproximou esta última dos serviços ofertados na unidade. As falas abaixo refletem esta análise:

"Uma das coisas mais marcantes é que os homens não procuravam a unidade [...] não sabiam da importância" (P9).

"Dois fatos marcantes[...] não conhecer o tratamento[...] participação da comunidade e da equipe para mudar mais a visão da linha de cuidado" (P8).

"Antes, oito pacientes cadastrados, depois cento e oitenta [...] mudou a questão do atendimento [...] projeto diabetes" (P10).

"Era marcante a resistência ao tratamento, mas melhorou [...] como também o acolhimento e envolvimento da equipe" (P12).

"A implantação do Programa de Saúde do Adolescente foi um espaço criado, que possibilitou aproximação com o adolescente" (P2).

"A alegria em participar dos fóruns e da Feira de Saúde" (P6).

Estes repertórios interpretativos também apontam para a fertilidade de práticas de saúde fundamentadas nos processos de conversação e negociação constantes entre todos os atores sociais envolvidos, neste caso, os profissionais de saúde da família, os estudantes, os docentes e os usuários dos serviços de saúde ${ }^{16,17}$.

A expressão do prazer dos participantes durante relações e vínculos produzidos nos leva a interpretar tais experiências como também produtoras de "encontros terapêuticos", tão necessários à produção de linhas de cuidado integral $^{6}$.

Facilidades e dificuldades do processo de construção de linhas de cuidado

No que diz respeito à questão sobre quais foram ou têm sido as facilidades na construção da linha de cuidado, verificou-se que os participantes citaram o PET-Saúde como elemento impulsionador dos seguintes aspectos do processo de trabalho em saúde da família: integração dos membros da equipe, melhoria do acolhimento aos usuários e aproximação com serviços da política de assistência social existentes na comunidade.

A integração de todos os recursos disponíveis faz parte de um agir comprometido com a construção da linha de cuidado ${ }^{15}$, tornando-se, portanto, facilitadora desse processo. Para exemplificar esta afirmativa, temos as falas a seguir:

"Equipe aceitou o trabalho [...] equipe PET envolvida [...] disponibilidade de ambos" (P1).

"A existência da linha de cuidado [...] acolhimento [...] alunos do PET" (P6).

"Integração da equipe [...] dos ACS [...] alunos do PET [...] e grupo de idosos" (P5).

"Integração das equipes [...] entrosamento com o PET [...] comunidade, por já desenvolver o trabalho há muito tempo" (P8).

"Apoio da igreja dos mórmons [...] igreja católica [...] casa do Pró-jovem [...] vínculo com as escolas [...] presença de outros profissionais, fisioterapeutas, nutricionista, dentista" (P8).

É notório o acolhimento como modalidade de intervenção que estabelece relação interpessoal mais próxima dos usuários em suas demandas, com isso, podendo organizar prática em saúde respeitando as crenças e os modos de agir do usuário. Ao se propor uma ação em saúde para determinada população, é importante que se esteja atento aos valores e crenças compartilhados pelo grupo ${ }^{9}$.

Em relação às dificuldades para a construção das linhas de cuidado, os conteúdos emergentes das falas dos participantes evidenciaram que problemas de gestão, déficit de trabalhadores de saúde, precarização do vínculo institucional, rotatividade de profissionais e violência na comunidade interferiam diretamente nas práticas das equipes, dificultando o processo de construção das linhas de cuidado. As falas abaixo evidenciam essas constatações:

"Não temos essa linha de cuidado implantada [...] saúde do homem [...] violência no bairro, teve que parar as entrevistas [...] microáreas descobertas [...] visitas aleatórias" (P9).

"Duas microáreas desassistidas [...] violência [...] gestão (unidade fechada) [...] funcionários desestimulados [...] falta de medicamentos" (P12). 
"Gestão (atraso dos salários) atrapalhou muito o trabalho, violência, dificuldade de acesso" (P2).

"Paralisações [...] áreas descobertas [...] implantação do Programa dentro do próprio município [...] estratégias para motivar a continuidade do Programa" (P5).

Retratamos, a partir do exposto acima, os sentidos acerca das noções que vêm embasando as propostas e a conduta dos gestores das políticas públicas locais frente aos problemas vivenciados pela população. Isto também demonstra os entraves para eficácia do trabalho das ESF's com a comunidade.

Essas constatações e a produção de sentidos aqui apresentada acerca da construção de linhas de cuidado descrevem as relações que compõem o cotidiano das equipes PET-Saúde e da USF no município estudado. Constatou-se que o cotidiano, atravessado por diversas práticas discursivas e por relações, contribuem para refletir os aspectos favoráveis e impeditivos, assim como os desafios para a (re) construção de linhas de cuidado no cenário da ESF $^{16,17}$.

A invenção de determinada configuração de mundo envolve alianças e oposições do que se pretende construir, entre os sujeitos que dele participam $^{11}$. Portanto, os desafios das relações aqui colocados se situam no contexto das condições precárias de trabalho, das adversidades do ambiente e da falta de acesso aos recursos necessários à produção do cuidado.

\section{Conclusões}

Os sentidos produzidos neste estudo evidenciaram singularidades e heterogeneidade do processo de construção das linhas de cuidado por equipes do PET-Saúde em Ilhéus-Bahia, tanto nas escolhas dos grupos prioritários ou do problema para intervenções, quanto nas trajetórias percorridas por cada equipe em seu contexto de atuação. Alguns consensos também foram identificados, mais relacionados à gestão do trabalho, no que diz respeito à qualidade das relações produzidas, aos ganhos na adesão aos serviços ofertados, e às aproximações com outros pontos da rede de cuidados, ou seja, com serviços da política da assistência social.

São notórios os problemas decorrentes da fragilidade e retrocessos do SUS, impedindo muitas vezes de traduzir aplicabilidade na prática das ações planejadas, evidenciando limites para a construção das linhas de cuidado, conforme proposto por cada equipe de trabalho. Contudo, é perceptível o reconhecimento dos participantes da pesquisa no que se refere à colaboração de vivências acadêmicas, a exemplo do PET-Saúde como ferramenta para produzir os cuidados à saúde, possibilitando buscar linhas de fuga (saídas) para enfrentar os limites e superar as dificuldades.

Os limites deste estudo dizem respeito aos seguintes aspectos metodológicos: tempo de coleta de dados, uma vez que não incluiu todo o período de atuação do PET-Saúde no município estudado; número de grupos focais, pois mais grupos poderiam trazer elementos textuais que possibilitassem enunciados complementares aos que foram aqui produzidos; não inclusão de atores dos grupos-alvo dos cuidados produzidos na condição de participantes do estudo. Diálogos com esses atores poderiam melhor explicitar aproximações e embates do processo de construção de linhas de cuidado.

Desta forma, este estudo contribui para refletir movimentos apreendidos de transformação do modelo de assistência centrado na doença, imprimindo olhares ampliados e busca de respostas às demandas do cidadão individual e da coletividade, os quais caracterizam o PET-Saúde e outros programas articuladores do ensino-serviço, como constitutivos de compromisso social e de qualificação dos serviços em saúde no território.

\section{Referências}

1. Santos $A M$, Kochergin $C N$, Jesus $S R$, Soares DA, Assis MMA, Bispo Júnior JP, et al. Linhas do cuidado e responsabilização no PSF de um município da região sudoeste da Bahia: um olhar analisador. In: Assis MMA, Nascimento MAA, Franco TB, Jorge MSB, organizadores. Produção do cuidado no Programa Saúde da Família: olhares analisadores em diferentes cenários. Salvador: EDUFBA; 2010. P. 59-82.

2. Gutierrez DMD, Minayo MCS. Produção de conhecimento sobre cuidados da saúde no âmbito da família. Ciênc. saúde coletiva 2010; 15(Suppl 1):1497-1508.

3. Ministério da Saúde (BR). Política Nacional de Humanização. Brasília: Ministério da Saúde; 2010.

4. Ogata MN, França Y. Atuação do auxiliar de enfermagem na Estratégia de Saúde da Família. Revista Acta Paul Enferm 2010; 23(4): 506-511.

5. Salgado MF, Pessôa LR, Franco $C$, Santos EHA, Torres KROB, Santos AS, organizadores. Manual do gerente: desafios da média gerência na saúde. Rio de Janeiro: ENSP; 2011.

6. Silva NEK, Sancho LG, Figueiredo WS. Entre fluxos e projetos terapêuticos: revisitando 
as noções de linha do cuidado em saúde e itinerários terapêuticos. Ciênc. saúde coletiva. 2016; 21(3): 843-852.

7. Marra AV, Brito VGP. Construcionismo Social e Análise do Discurso: Uma possibilidade teórico-metodológica. In: Anais do XXXV Encontro da Associação Nacional de PósGraduação e Pesquisa em Administração, 2011; Rio de Janeiro; 2011.

8. Bosi MLM, Sousa MAM. Anotações sobre a análise crítica de discurso em pesquisas no campo da saúde. Rev. Bras. Saude Mater. Infant. 2014; 14: 423-432.

9. Spink MJ. As múltiplas faces da pesquisa sobre produção de sentidos no cotidiano. In: Spink MJ. Linguagem e produção de sentidos no cotidiano; Rio de Janeiro: Centro Edelstein de Pesquisas Sociais; 2010. P. 38-59.

10. Campos-Fonseca L. Estudo de caso dos repertórios interpretativos empregados na construção de posicionamentos contrários ao sistema de cotas raciais nas universidades públicas brasileiras em comentários na internet. [Dissertação de Mestrado]. São Paulo: Instituto de Psicologia da Universidade de São Paulo IP/USP; 2014.

11. Rocha D. Representar e intervir: linguagem, prática discursiva e performatividade. Linguagem em (Dis)curso 2014; 14(3): 619-632.

12. Brasil. Conselho Nacional de Saúde. Resolução no 466 de 12 de dezembro de 2012. Trata de pesquisas em seres humanos e atualiza a resolução 196 [Internet]. Brasília, DF; 2012. [acesso em 2018 ago. 10]. Disponível: http://conselho.saude.gov.br/resolucoes/2012/R eso466.pdf

13. Osmo A, Schairaber L. O campo da Saúde Coletiva no Brasil: definições e debates em sua constituição. Saude Soc 2015; 24: 201-214.

14. Bertotti AP, Lenzi MCR, Portes JRM. O portador de Esclerose Múltipla e suas formas de enfrentamento frente à doença. Barbaroi 2011; 34: 101-124.

15. Dubow C, Olivo VMF, Ceron MIVDO, Dal Moro JS, Oliveira CP et al. Linha de cuidado como dispositivo para a integralidade da atenção a usuários acometidos por agravos neoplásicos de cabeça e pescoço. Saúde debate. 2014; 38(100): 94-103.

16. Mendes EV. As redes de atenção à saúde. 2. ed. Brasília: Organização PanAmericana da Saúde; 2011.

17. Fertonani HP, Pires DEP, Biff D, Scherer MDA. Modelo Assistencial em Saúde: conceitos e desafios para a atenção básica brasileira. Ciênc. saúde coletiva $2015 ; 20(6):$ 1869-1878.

\section{Endereço para Correspondência}

Universidade Estadual de Santa Cruz - UESC Rodovia Jorge Amado, Km 16, Bairro Salobrinho Ilhéus/BA

CEP.: 45662-900

e-mail: meirenubia@yahoo.com.br

Recebido em 18/11/2017

Aprovado em 06/09/2018

Publicado em 12/09/2018 